\title{
Modeling the evolution of the aerosol cloud of toxicants in the atmosphere
}

\author{
Ivan Bondarchuk ${ }^{1}$, Sergei Bondarchuk ${ }^{2}$, and Boris Borisov , $^{3}$ \\ ${ }^{1}$ Tomsk State University, 634050 Tomsk, Russia \\ ${ }^{2}$ Tomsk State Pedagogical University, 634061 Tomsk, Russia \\ ${ }^{3}$ Tomsk Polytechnic University, 634050 Tomsk, Russia
}

\begin{abstract}
Using the methods of mathematical modeling, the formation and evolution of aerosol clouds of toxicants in the atmosphere from the chemical industry enterprises, thermal power engineering and rocket carriers of space vehicles is analyzed. The processes of dynamic interaction of drops between themselves and a two-phase flow, processes of agglomeration, crushing and evaporation of aerosol particles are taken into account. The results of numerical calculations are presented.
\end{abstract}

\section{Introduction}

Increasing the impact of human life on the environment requires constant improvement of the means of monitoring this impact and analyzing its consequences. One of the most important objects of the described impact influence is the atmosphere. It is accepts the toxicants flow ejected from the pipes of thermal power plants, chemical plants, etc. of. A significant contribution to the violation of the environment is made by space vehicles and aircraft, especially when forcibly dumping the remains of fuel components before a forced, unforeseen or emergency landing of the apparatus.

The present work is devoted to mathematical modeling of the formation, evolution and distribution of toxic aerosols in the atmosphere with the localization of the site of their deposition, the analysis of the possible degree of contamination for a particular area, taking into account various physical and meteorological factors in order to predict possible environmental risks. When formulating a mathematical model, the features of the processes of dynamic interaction of moving deformable particles (droplets) of a dispersed phase in the general case with a two-phase dispersion medium are considered [1-5]; the influence of meteorological features of the region is analyzed; the set of interrelated processes deformation and fragmentation of drops with the analysis of processes of their evaporation and heat and mass exchange with a turbulent atmosphere [6-7] is taken into account. Reviews on this problem are presented in [8-9].

The paper presents the results of mathematical modeling of the evolution and dynamics of a liquid-drop cloud in the case of an emergency dump of liquid aviation or rocket fuel.

\footnotetext{
*Corresponding author: bvborisov@tpu.ru
} 


\section{Physical and mathematical models of processes}

As shown by the analysis of the experimental data [10] in the range of moderate $(\operatorname{Re} \approx 10 \div 100)$ and small $(\operatorname{Re} \leq 1)$ Reynolds numbers in the region of large Bond numbers Bo typical for the aerosol droplets under consideration, the deformation of the droplet from the Weber number We [11] is determined by the relation:

$$
\varepsilon=\frac{D_{m}}{D_{0}}=1+0.027 \mathrm{We},
$$

where $D_{m}$ is the diameter of the average cross section of the drop; $D_{0}$ - diameter of an equivalent spherical drop (whose volume is equal to the volume of the deformed drop).

The similarity criteria for the conditions of specific experiments using the following formulas were calculated:

$$
\operatorname{Re}=\frac{\rho\left|\boldsymbol{U}-\boldsymbol{U}_{p}\right| D}{\mu}, \quad \mathrm{We}=\frac{\rho\left|\boldsymbol{U}-\boldsymbol{U}_{p}\right|^{2} D}{\sigma}, \quad \text { Bo }=\frac{\rho_{p} D^{2}}{\sigma}|\boldsymbol{\omega}|,
$$

where $\sigma$ is the coefficient of surface tension; $\rho_{\mathrm{p}}$ is the density of the drop.

The process of coagulation and fragmentation of aerosol particles on accordance with the generalized approximation formula for the collision parameter of droplets of a twophase flow of similar sizes $[11,12]$ was modelled:

$$
\Phi_{j i}=1.4+1.979 \beta+0.507\left(2 \beta^{2}-1\right)
$$

where $\beta=\left(\frac{\mathrm{Re}_{j i}}{383.6}\right)^{0.572}\left(\frac{\mathrm{Lp}_{i}}{370.37}\right)^{-0.153}\left(\frac{\gamma_{j i}}{2.37}\right)^{-0.597} ; \Phi_{j i}$ is the mathematical expectation of the ratio of the change in the mass of the target drop $D_{i}$, caused by the interaction of $j-i$, to the total mass of the projectile droplets $D_{j}\left(D_{j}<D_{i}\right)$ that collide with it. $\operatorname{Re}_{j i}=6$ to 385; $\mathrm{Lp}_{i}=0.2$ to $600 ; \operatorname{Re}_{j i}=1 \div 12 ; \operatorname{Re}_{j i}$ - Reynolds number, calculated by the diameter of the drop-projectile $D_{j}$; Lp is the Laplace number; $\gamma_{j i}$ is the ratio of the diameters of the colliding drops.

The Reynolds number from the diameter of the $D_{j}$ projectile was calculated. Equations of motion of a droplet of radius rp for the its velocity componens in a Cartesian coordinate system relative to the surface (the $\mathrm{z}$ axis is directed along the gravitational acceleration vector of gravity of gravity) has the form [11]:

$$
\frac{d u_{p}}{d t}=\varphi_{1}\left(u-u_{p}\right), \frac{d v_{p}}{d t}=\varphi_{1}\left(v-v_{p}\right), \frac{d w_{p}}{d t}=\varphi_{1}\left(w-w_{p}\right)+g,
$$

where $\varphi_{1}=\frac{3 \rho}{8 \rho_{p} \boldsymbol{r}_{p}} \boldsymbol{C}_{\boldsymbol{D}}\left|\boldsymbol{U}-\boldsymbol{U}_{p}\right| ;\left|\boldsymbol{U}-\boldsymbol{U}_{\boldsymbol{p}}\right|=\sqrt{\left(\boldsymbol{u}-\boldsymbol{u}_{p}\right)^{2}+\left(\boldsymbol{v}-\boldsymbol{v}_{p}\right)^{2}+\left(\boldsymbol{w}-\boldsymbol{w}_{p}\right)^{2}} ; u, v, w$ are the components of the wind speed vector $\boldsymbol{U} ; u_{p}, v_{p}, w_{p}$ are the components of the velocity vector of the droplet $\boldsymbol{U}_{p} ; C_{D}$ is the coefficient of aerodynamic resistance.

At $\operatorname{Re}=(1 \div 700)$ (intermediate flow regime), the Klyachko formula to determine the coefficient of aerodynamic drag was used [13]: 


$$
C_{D}=\frac{24}{\operatorname{Re}}+\frac{4}{\sqrt[3]{\mathrm{Re}}}
$$

For $\operatorname{Re}=(700 \div 3 \cdot 105)$ (self-similar flow) $[14], C_{D}=0.44$ was assumed. The process of aerodynamic crushing of droplets was determined in accordance with the calculated values of the Weber and Bond criteria. In accordance with the analysis of the experimental data, it was assumed that when the critical Weber number $\mathrm{We}=\mathrm{We}^{*}=17$ or the critical value of the Bond number is $\mathrm{Bo}=\mathrm{Bo} *=22.5$, the drop is split into two spherical drops of equal mass. The physical properties of air due to vertical coordinate of account for a specific date using the parameters of the standard atmosphere and the data of weather stations was taken on. The coefficient of dynamic viscosity of air using the Sutherland formula [15] was calculated:

$$
\mu=\frac{0.68 \cdot 10^{-2}}{T+122}\left(\frac{T}{273}\right)^{3 / 2},
$$

where $T$ is the absolute temperature.

The drop is a thermally thin body, and its temperature averaged over the volume $T_{p}$ in accordance with the following equation was determined:

$$
\frac{d T_{p}}{d t}=\varphi_{2} \operatorname{Nu}\left(T-T_{p}\right)-\frac{q_{r}}{m c_{p}} G,
$$

where $\varphi_{2}=\frac{3}{2} \frac{\lambda}{r_{p}^{2} \rho_{p} c_{p}} ; c_{p}$ is the specific heat of the liquid; $\lambda$ is the coefficient of thermal conductivity of air; $m$ is the mass of the drop; $G$ is the mass of the evaporated liquid from the drop surface per unit time (evaporation rate); $q_{r}$ is the specific heat of evaporation of the liquid.

To calculate the Nusselt number, the following dependence was used [16]: $\mathrm{Nu}=2+0.6 \operatorname{Re}^{1 / 2} \operatorname{Pr}^{1 / 3}$, where $\operatorname{Pr}$ is the Prandtl number. The change in the droplet radius due to evaporation on the equation was determined:

$$
\frac{d r_{p}}{d t}=-\frac{G}{4 \pi \rho_{p} r_{p}^{2}}
$$

The evaporation rate by the formula was determined:

$$
G=4 \pi r_{p}^{2} k \frac{X}{1-X}
$$

where $k$ is the mass-transfer coefficient; $\mathrm{X}$ is the mole fraction of the vapor near the drop surface.

Equation (7) in accordance with the relationship between $\mathrm{X}$ and the partial vapor pressure $p_{0}$ was written:

$$
\frac{d r_{p}}{d t}=-\frac{k}{\rho_{p}} \frac{p_{0}}{p-p_{0}},
$$

where $p$ is the atmospheric pressure. The mass-transfer coefficient $\mathrm{k}$ from the formula [17] was calculated: 


$$
k=\frac{c_{f} D_{f} M_{p}}{2 r_{p}}\left[2+0.6\left(\frac{2 r_{p}\left|\boldsymbol{U}-\boldsymbol{U}_{p}\right| \rho_{f}}{\mu_{f}}\right)^{1 / 2}\left(\frac{\mu_{f}}{\rho_{f} D_{f}}\right)^{1 / 3}\right],
$$

where $D_{f}$ is the binary diffusion coefficient at the film temperature $T_{f}=\left(T_{f}+T\right) / 2 ; c_{f}$ and $\mu_{f}$ are the density and coefficient of dynamic viscosity of air at $T_{f}$.

The diffusion coefficient in accordance with the Fuller-Schletter-Giddings [18] proposals for binary gas systems at low pressure was calculated:

$$
D=\frac{T^{1.75}\left[\left(M_{A}+M_{B}\right) /\left(M_{A} M_{B}\right)\right]^{0.5}}{p\left[\left(\sum V_{A}\right)^{1 / 3}+\left(\sum V_{B}\right)^{1 / 3}\right]^{2}},
$$

where $\left[D_{f}\right]=\mathrm{cm} 2 / \mathrm{sec} ;[p]=\mathrm{atm} ;[T]=\mathrm{K} ; M_{A}$ and $M_{B}$ are the molecular masses of components $\mathrm{A}$ and $\mathrm{B}$ (drops of liquid and air); $\sum V_{A}, \sum V_{B}$ are the diffusion volumes of molecules.

The trajectory of droplet motion was determined in accordance with kinematic equations:

$$
u_{p}=\frac{d x}{d t}, v_{p}=\frac{d y}{d t}, w_{p}=\frac{d z}{d t}
$$

\section{Simulation results}

For numerical integration of the system (4) - (12), the implicit difference scheme for the linearized right-hand sides was used [11]. In the calculations, the dynamics of each droplet fraction with a fixed size in the range $0-3 \mathrm{~mm}$ was separately analysed. At each time step, the kinematics of the motion is separately considered, taking into account the possibility of aerodynamic fragmentation of the droplet, the new droplet coordinates and the components of its velocity vector are determined, the new droplet temperature is determined, and finally, the droplet size are modelled taking into account evaporation. Integration of the system of equations for each fraction until the droplet evaporates completely or when it reaches, the surface of the Earth is carried out. The reliability of the results of numerical modelling of the formation and the dynamics of the change in aerosol clouds of toxicants in the atmosphere comparison with typical experimental data of the results of the launch of the "Proton" carrier rocket on October 26, 2007 [10] was verified by. In this case, the toxicant was an asymmetric dimethyl hydrazine (component of liquid fuel), and the height of the formation of the toxic aerosol cloud was about $30103 \mathrm{~m}$. The projections of the trajectories of aerosol particles of the main calculated fractions shows in Fig. 1. The experimentally confirmed discrepancy between the trajectories of different particle sizes the influence of the wind speed varying in altitude was explained by.

The change in the rate of evaporation of particles in height corresponds due to the change of the atmospheric temperature on altitud (Fig. 2).

Figure 2 shows the rate of evaporation $G$ in height, defined as the mass of evaporated toxicant per meter of fall height and the corresponding atmospheric temperature $t$. Completely evaporation of drops of a maximum initial size of $3 \mathrm{~mm}$ took place at an altitude of $2200 \mathrm{~m}$. Good qualitative and satisfactory quantitative coincidence of the calculations and analysis contained in [10] allows us to recommend the calculation methodology developed by the authors for its use in assessing the impact of thermal energy facilities, chemical plants, etc. on the environment. 


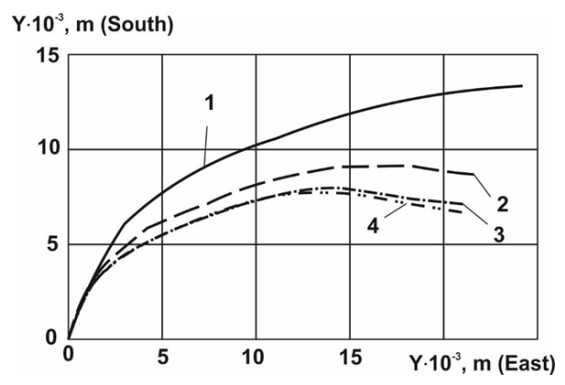

Fig. 1. Projections of trajectories of particles of different initial radius on a horizontal plane. $2 \mathrm{~mm}$ curve number $1 ; 3 \mathrm{~mm}$ - curve number $2 ; 1.5$ - curve number $3 ; 1 \mathrm{~mm}$ - curve number 4 .

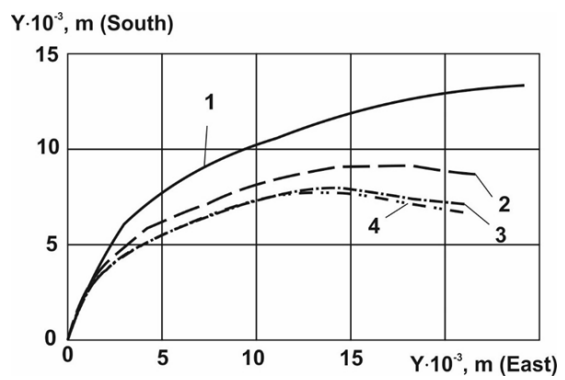

Fig. 2. Mass evaporation rate $\mathrm{G}$ and temperature of the atmosphere $t$ in height.

Ministry of Education of Russia sponsored the study within the base part No. 6.8356.2017.

\section{References}

1. G. K. Batchelor, An introduction to fluid dynamics (Cambridge Univ. Press, London and New York, 1967)

2. G. Hetsroni, Handbook of multiphase systems (Hemisphere Publishing Corporation, Washington, New York, London, 1982)

3. J. F. Brady, G. Bossis, Annu. Rev. Fluid. Mech., 20, 1 (1988), DOI: 10.1146/annurev.fl.20.010188.000551

4. C.T. Crowe, R. Johnson, A. Prosperetti, M. Sommerfeld, Y. Tsuji, Society of Mechanical Engineers, 236, 1 (1996)

5. J.D. Schwarzkopf, C.T. Crowe, P. Dutta, AIChE Journal, 55, 6 (2009), DOI: 10.1002/aic. 11773

6. V.I. Terekhov, V.V. Terekhov, N.E. Shishkin, K.Ch. Bi, J. Eng. Phys. Thermophys., 83, 5 (2010), DOI: 10.1007/s10891-010-0410-7

7. M.A. Pakhomov, V.I. Terekhov, International Journal of Heat and Mass Transfer, 66, 210-222 (2013), DOI: 10.1016/j.ijheatmasstransfer.2013.07.013

8. E.L. Aleksandrov, Meteorol. Hydrol., 4, 36-45 (1993)

9. E.L. Aleksandrov, Behavior of liquid rocket fuels in atmosphere (ANKIL, Moscow, 2000)

10. V.A. Arxipov, E.A. Kozlov, S.S. Titov, A.S. Tkachenko, A.S. Usanina, I.K. Zharova, Arab. J. Geosci., 9, 2 (2016), DOI: 10.1007/s12517-015-2161-4

11. I.M. Vasenin, V.A. Arkhipov, V.G. Butov, A.A. Glazunov, V.F. Trofimov, Gas dynamics of two-phase flows in orifices (TSU, Tomsk, 1986) 
12. V. A. Arkhipov, V.G. Butov, I.M. Vasenin, F.G. Gaponich, A.M. Podvysotsky, V.F. Trofimov, A.A. Shrayber, App. Mech. Appl. Phys., 5, 90-94 (1985)

13. L.S. Klyachko, Otoplen. Ventil., 4, 27-29 (1934)

14. R. Clift, J.R. Grase, M.E. Weber, Bubbles, drops and particles (Acad. Press., New York, 1978)

15. G.N. Abramovich, Applied gas dynamics (Nauka, Moscow, 1991)

16. S.K. Aggarwal, AI. Tong, W.A. Sirignano, AIAA Journal, 22, 1448-1457 (1985), DOI: $10.2514 / 3.8802$

17. R.B. Bird, W.E. Stewart, E.N. Lightfoot, Transport Phenomena (John Wiley and Sons, New York, 1974)

18. R.C. Reid, J.M. Prausnitz, T.K. Sherwood, The properties of gases and liquids (Chemistry, Leningrad, 1982) 